\title{
La cybercommunication et son intégration dans le plan stratégique
}

\author{
William Menvielle, Denis Pettigrew et Jocelyn D. Perreault ${ }^{1}$ \\ Université du Québec à Trois-Rivières
}

\section{Introduction}

Cet article est le dernier de la série mettant en lumière l'impact des nouvelles technologies sur le marketingmix. Ainsi, après avoir analysé les enjeux du produit, du prix et de la distribution, nous nous attaquerons à développer les aspects de la communication électronique $^{2}$.

Précisons d'abord que la communication sur Internet ne se limite pas exclusivement à la publicité. Cet amalgame, utilisant les termes communication et publicité de façon interchangeable, est aussi un constat souvent fait dans le cybermonde. Une des explications est sans doute que la publicité électronique est la forme de communication électronique la plus visible par le grand public. Ainsi, le but de cet article est de présenter la communication électronique sous l'angle de la communication marketing intégrée et de définir les atouts et l'utilisation des différents outils de la communication marketing.

Bien que nous ne parlerons que des aspects tactiques de la communication, il convient de présenter la place de cette dernière dans la stratégie de marketing et de focaliser sur les dimensions étudiées ici. La figure 1 de la page suivante reproduit le processus de la planification stratégique en marketing qui comprend la variable communication ${ }^{3}$. Elle correspond à la dimension étudiée dans cet article, tout en mettant principalement l'accent sur les outils de la communication.

Il ne sera pas question de présenter les stratégies à proprement parler, mais bien plus de voir en détail les outils traditionnels et électroniques de la communication marketing intégrée utilisés par les entreprises.

\section{La communication marketing intégrée}

De la communication à la communication marketing intégrée, il n'y a qu'un pas, du moins semble-t-il. Pour définir le concept de communication, nous retenons une définition se posant comme une référence en la matière et qui s'énonce comme suit : "La communication marketing est l'ensemble des signaux émis par l'entreprise en direction de ses différents publics, c'est-à-dire auprès de ses clients, distributeurs, fournisseurs, actionnaires, auprès des pouvoirs publics et également vis-à-vis de son propre personnel $\gg{ }^{4}$.

De son côté, la communication marketing intégrée est un concept d'importance qui a soulevé un grand intérêt au cours des dernières années. La désintégration des marchés de grande consommation, la multiplicité des micro-marchés, la prolifération des nouveaux outils de communication et les modifications du comportement du consommateur poussent l'entreprise à repenser son choix et son utilisation des outils de communication. Cette tendance s'inscrit dans ce que l'on nomme la Communication marketing intégrée (CMI), et qui se définit comme suit : " IMC is a concept of marketing communications planning that recognizes the added value of a comprehensive plan that evaluates the strategic roles of a variety of communication disciplines (for example general advertising, direct response, sales promotion and public relation)... and combines these disciplines to provide clarity, consistency, and maximum communication impact $» 5$. 
Figure 1 - Processus de planification stratégique en marketing

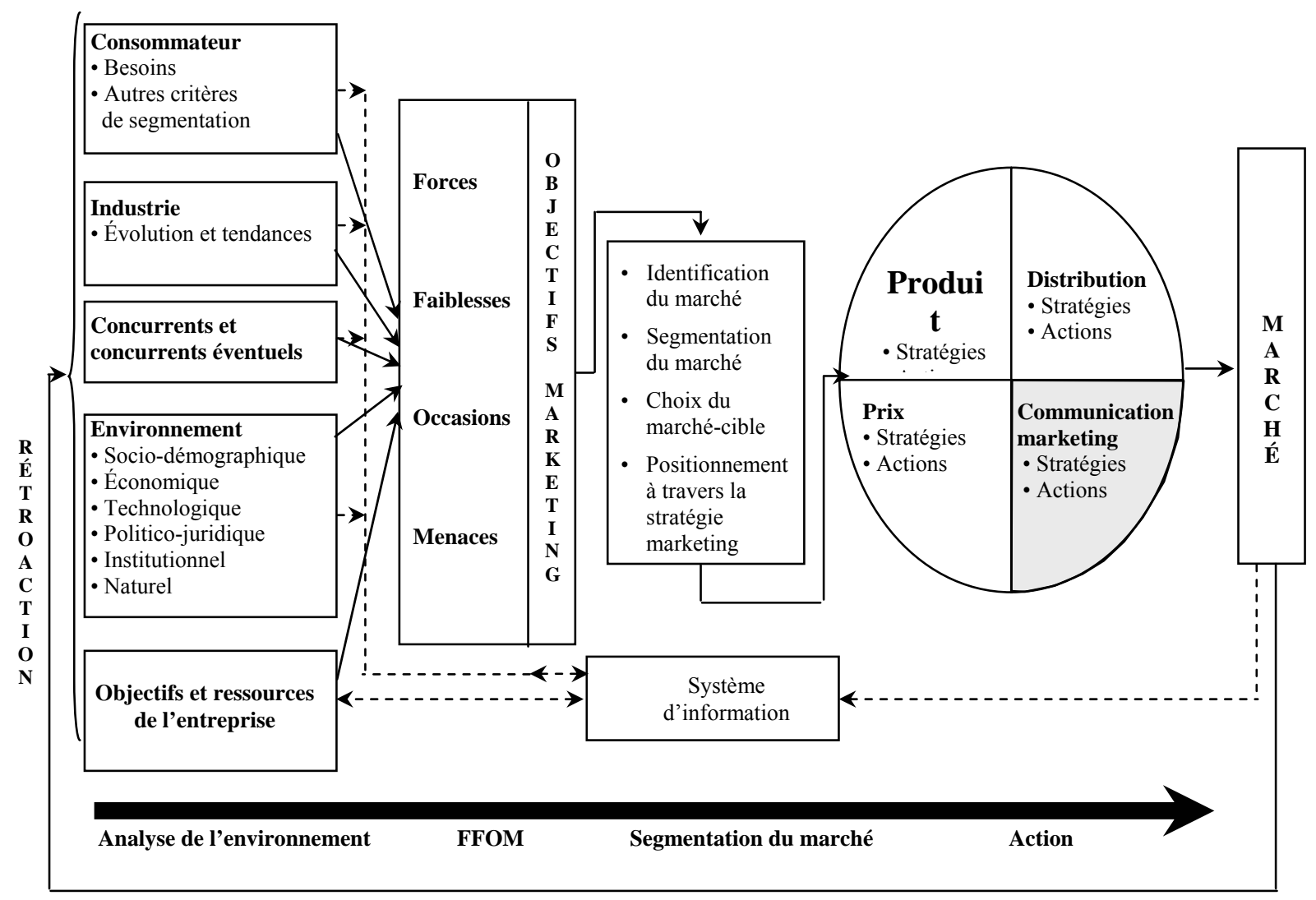

Cette définition inclut implicitement la notion de planification stratégique et de coordination de divers outils de communication marketing propres à une entreprise. À la lumière des propos tenus lors de la définition de la communication marketing intégrée, il y a consensus pour dire que les principaux outils du mix de communication se composent de la publicité, de la promotion des ventes, des relations publiques, du marketing direct et de la force de vente ${ }^{6}$.

Il subsiste toutefois une mésentente lorsqu'il est question de l'Internet. S'il ne fait pas de doute qu'il se positionne comme le sixième outil, il n'en demeure pas moins qu'il est difficile à intégrer en raison de ses caractéristiques spécifiques : interactivité, interconnectivité, instantanéité, variété des outils de communication qu'il couvre et remplace.

Rares sont les auteurs ayant proposé une façon d'intégrer Internet aux autres outils précédemment cités. Une de ces classifications fait état de trois façons d'intégrer Internet aux autres outils de communication :
- comme un outil de bas niveau : dans ce cas, Internet est à considérer comme un soutien aux activités traditionnelles de communication;

- comme sixième outil à part entière : Internet est considéré sur le même pied d'égalité que tous les autres outils;

- comme outil suprême de communication : Internet a préséance sur toutes les autres formes de communication. L'outil est utilisé en priorité avant tous les autres ${ }^{7}$.

En fait, si l'on y regarde d'un peu plus près, on constate que les outils de la communication traditionnelle ont tous leur pendant sur Internet. La figure 2 dresse le portrait des liens pouvant être établis entre les outils de communication traditionnelle et électronique.

Les outils présentent des liens allant de la communication traditionnelle à la communication électronique. En fait, nous pensons que la communication électronique ne remplace pas la communication traditionnelle : ces deux formes de communication se com- 
plètent, elles convergent. Dans cette situation, il existe le double électronique de l'outil traditionnel. Par exemple, on retrouve les mêmes outils dans l'une ou l'autre des formes de communication: relations publiques dans le monde traditionnel et relations publi- ques virtuelles dans le monde électronique, coupons traditionnels et électroniques, etc. Il s'agit ainsi d'une forme d'intégration puisque la plupart des outils traditionnels ont été incorporés dans Internet.

Figure 2 - Les liens entre les outils des communications traditionnelle et électronique

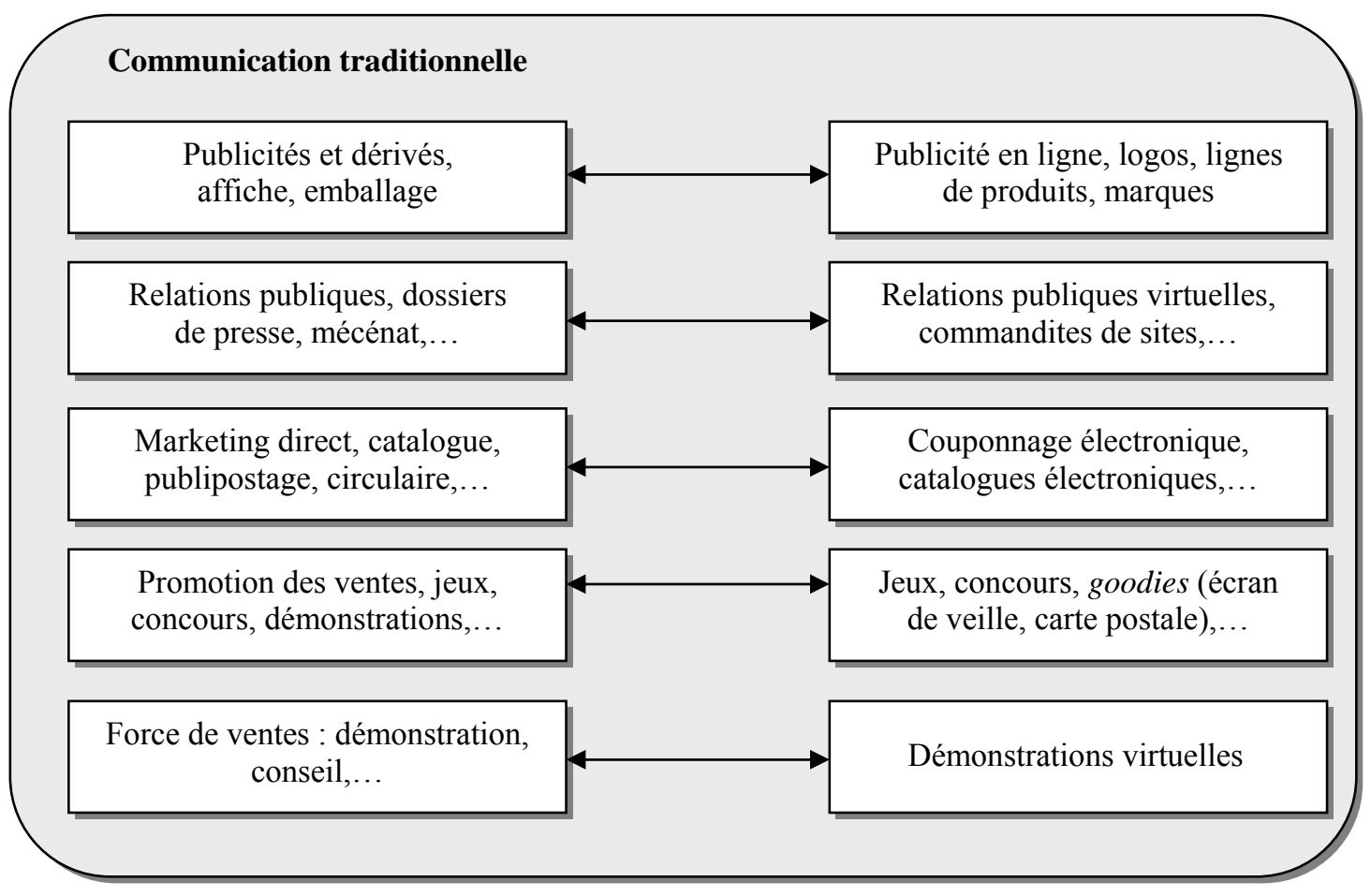

Nous proposons ainsi d'explorer les quatre principaux outils de la communication marketing intégrée (publicité, relations publiques, marketing direct et promotion des ventes) afin de comprendre comment ils ont été incorporés dans la communication électronique, quelles entreprises les utilisent et dans quelle proportion.

Pour illustrer nos propos, nous nous sommes basés sur des études canadiennes et américaines. Pour ces dernières, les mêmes auteurs ont effectué des analyses, en partie descriptives, afin de dresser le portrait de divers types d'entreprises et de secteurs d'activité. Ainsi, certains ont dressé le portrait de 100 détaillants ayant un site $\mathrm{Web}^{8}$, tandis que la recherche de leurs collègues présente 152 détaillants de la base d'entreprises américaines Fortune $500{ }^{9}$. Pour les auteurs Canadiens, c'est une vaste étude à partir de données secondaires principalement qui a été effectuée de façon longitudinale auprès de plus de 5000 sites Web au Québec ${ }^{10}$.

\section{Les outils de la communication marketing électronique}

\section{La publicité électronique}

De façon traditionnelle, la publicité média est « la communication diffusée, après achat publicitaire, dans les grands médias (presse, télévision, radio, cinéma et affichage) $\gg{ }^{11}$. Dans le cyberespace, cet outil joue le même rôle que dans le monde physique : il vise à attirer l'attention, à fournir de l'information, à positionner une marque et, dans une moindre mesure, à inciter à l'essai et à pousser les consommateurs à l'action. Quoi qu'il en soit, il semble utile de préciser la particularité de la publicité sur Internet de la façon suivante: "Certaines formes de publicité peuvent en effet être considérées comme de la publicité au sens où l'on l'entend traditionnellement dans les médias classiques. D'autres formes de publicité sont plus proches des outils du hors média et du marketing direct. 
Enfin, il existe des formes de publicité sur Internet qui ne sont classables dans aucune des deux catégories précédentes. Internet engendre donc une nouvelle catégorie de publicité qui lui est propre ${ }^{12}$.

\section{D'autres formes de publicité sont plus proches des outils du hors média et du marketing direct.}

Bien souvent, on entend par publicité Web des bandeaux dynamiques, parfois interactifs et visibles sur de nombreux sites d'information, des portails, des moteurs et des annuaires de recherche ou encore présents au sein des boîtes de courriers électroniques gratuits. Ces formes diverses ont évolué et se sont multipliées, donnant naissance aux bandeaux gratteciel, aux boutons, aux îlots, voire aux «pop-up » et aux interstitiels. Le but avoué était le même: faire cliquer le consommateur pour l'attirer vers le site de l'annonceur par un lien hypertexte. Toutefois, avec le temps et l'apprentissage de l'internaute, le taux de clic a chuté, rendant désuètes ces formes de publicité pourtant encore fort présentes. L'autre désavantage est que, pour être efficaces, elles doivent cibler les bons consommateurs, et sur des périodes de temps bien définies. Comme le taux d'efficacité des bandeaux traditionnels avoisine les $0,5 \%$, il ne semble pas intéressant pour une petite entreprise d'investir dans cet outil, sauf si elle opte pour une personnalisation des messages affichés, auquel cas le taux de clic avoisine les $10 \%$.
Toutefois, l'entreprise - même la PME - qui possède un site Web pourrait faire davantage de publicité en communiquant plus d'informations sur les produits, les prix ou la distribution, de même que de l'information corporative. L'information sur produit peut comprendre plusieurs dimensions comme la marque, le logo et les caractéristiques du produit. Un site purement informationnel peut donc contenir ces éléments. Le tableau 1 reproduit les principales composantes de la variable publicité telle que définie par les auteurs des études mentionnées en introduction. Plusieurs constats sont à faire. Tout d'abord, on note, pour le Québec, une forte proportion d'entreprises, toutes tailles et tous secteurs confondus, disposant de leur logo sur leur site Web.

Par ailleurs, pour les États-Unis, les différences sont notables selon les secteurs d'activités. L'étude de 2002 rapporte le constat auprès de manufacturiers, tandis que celle de 2003 se base sur les entreprises du commerce de détail. Une des explications que nous apportons est qu'un site Web est inutile pour une entreprise du commerce de détail s'il n'est pas transactionnel. Or, dans le commerce de détail, on trouve de nombreux petits détaillants, qui ont suivi la mode en bâtissant un site Web, mais avec un contenu parfois fort pauvre. De plus, ils distribuent souvent un grand nombre de produits de marques variées et nombreuses; il semble difficile de toutes les mentionner sur leur site Web. À l'inverse, pour les entreprises manufacturières, le site Web constitue une façon de trouver des partenaires d'affaires : se mettre en valeur est donc un atout.

\section{Tableau 1 - Synthèse des composantes de la publicité sur les sites Web des entreprises nord-américaines}

\begin{tabular}{|c|c|c|c|c|c|c|}
\hline & Composantes & $\begin{array}{c}\text { Perry et } \\
\text { Bodkin } \\
\text { (2003) }\end{array}$ & $\begin{array}{c}\text { Perry et } \\
\text { Bodkin } \\
\text { (2002) }\end{array}$ & $\begin{array}{c}\text { Bégin et } \\
\text { Boisvert } \\
\text { (2002) }\end{array}$ & $\begin{array}{c}\text { Perry et } \\
\text { Bodkin } \\
(2000)\end{array}$ & $\begin{array}{c}\text { Griffith et } \\
\text { Krampf } \\
\text { (1998) }\end{array}$ \\
\hline \multirow{3}{*}{ صَّ } & Logos & $31,9 \%$ & $92,0 \%$ & $83,5 \%$ & n.é. ${ }^{13}$ & n.é. \\
\hline & Lignes de produits & $17,0 \%$ & $58,0 \%$ & n.é. & n.é. & n.é. \\
\hline & Marques & $8,9 \%$ & $29 \%$ & n.é. & n.é. & n.é. \\
\hline
\end{tabular}

\section{Les relations publiques}

Les relations publiques sont constituées par «l'ensemble des moyens de communication utilisés par une entreprise pour créer un climat favorable dans les milieux avec lesquels elle est en rapport ${ }^{14}$. Leurs objectifs sont multiples, mais le principal est l'amélioration de l'image de l'entreprise. Dans certains cas, 
il s'agit également d'une amélioration de la notoriété de l'entreprise (lors du lancement d'un nouveau produit, par exemple), voire de créer un climat général de confiance.

Elles représentent un ensemble de moyens utilisés par les entreprises pour créer un climat de confiance auprès de leur personnel, dans les milieux dans lesquels elles sont en rapport et généralement dans le public en vue de soutenir leur activité et de favoriser leur développement. À ce chapitre, les moyens les plus couramment utilisés sont les dossiers de presse, les communiqués de presse, de l'information générale sur l'entreprise, les causes sociales soutenues par l'entreprise, le cours des actions, etc. Le tableau 2 dresse le portrait des composantes des relations publiques recensées dans diverses études auprès d'entreprises nord-américaines.

Cette fois encore, les chiffres démontrent une certaine variété selon les outils des relations publiques. L'étude auprès d'entreprises du commerce de détail montre des taux d'adoption assez élevés de ces outils, mais c'est aussi l'étude la plus récente parmi celles présentées ici. Notons aussi que ces dimensions doivent être mises en contexte : il faudrait savoir quelles sont les cibles des sites Web, ces derniers s'adressant généralement à différentes clientèles (consommateur final, investisseur, partenaires d'affaires, détenteurs d'enjeux), ce qu'aucune étude n'a mis en valeur.
De plus, cette variable présente des outils de nature fort différente sur le plan de l'instantanéité. Alors que les rapports annuels ne sont déposés qu'une fois l'an, la cotation des actions nécessite une mise à jour en continu. Au Québec, certaines entreprises comme Bombardier (www.bombardier.com) mettent à jour, aux trente minutes, le cours de leurs actions. Cette composante est une véritable "épée de Damoclès " sur la tête des entreprises parce que l'incapacité de fournir une information viable jette un discrédit sur l'entreprise.

Enfin, et mis à part la rubrique « information générale ", les entreprises québécoises semblent accuser un retard par rapport aux entreprises américaines, dont les études ont été menées de façon antérieure et postérieure à l'étude québécoise. Notons toutefois l'utilisation avant-gardiste de certains de ces outils, comme les communiqués de presse, utilisés efficacement par la PME Maax (www.maax.com), fer de lance dans les cabines de douche et les baignoires, ou encore le forum de discussion intégré à même le site Web. C'est le cas de l'entreprise de rénovation Réno-Dépôt (www.renodepot.com). Ce concept est innovateur puisqu'il crée une communauté virtuelle de passionnés, mais l'entreprise s'expose aux critiques et aux doléances des clients. C'est d'ailleurs ce qu'a vécu Volvo il y a plusieurs années, en décidant de fermer son forum de discussion à la suite de critiques du type : «Bon site Web, mais le toit ouvrant de ma 850 fuit ${ }^{16}$.

\section{Tableau 2 - Synthèse des composantes des relations publiques sur les sites Web des entreprises nord-américaines}

\begin{tabular}{|c|c|c|c|c|c|c|}
\hline & Composantes & $\begin{array}{l}\text { Perry et } \\
\text { Bodkin } \\
(2003)\end{array}$ & $\begin{array}{l}\text { Perry et } \\
\text { Bodkin } \\
(2002)\end{array}$ & $\begin{array}{c}\text { Bégin et } \\
\text { Tchokogué } \\
\text { (2001) }\end{array}$ & $\begin{array}{l}\text { Perry et } \\
\text { Bodkin } \\
(2000)\end{array}$ & $\begin{array}{c}\text { Griffith et } \\
\text { Krampf } \\
\text { (1998) }\end{array}$ \\
\hline \multirow{7}{*}{ 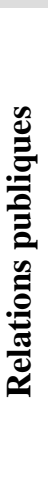 } & Information générale & n.é. & $39,6 \%$ & $\begin{array}{l}\text { De } 9,3 \% \text { à } \\
67,5 \% 15\end{array}$ & $22,74 \%$ & $67,2 \%$ \\
\hline & $\begin{array}{l}\text { Information à desti- } \\
\text { nation des actionnaires }\end{array}$ & $51 \%$ & $35,3 \%$ & n.é. & $15,88 \%$ & n.é. \\
\hline & Information financière & $34 \%$ & $30,9 \%$ & $2,4 \%$ & $11,55 \%$ & n.é. \\
\hline & Cours des actions & $20 \%$ & $19,6 \%$ & $1,4 \%$ & $10,11 \%$ & n.é. \\
\hline & Revue de presse & $38 \%$ & $20,5 \%$ & $2,5 \%$ & $8,66 \%$ & $54,69 \%$ \\
\hline & Rapports annuels & $20 \%$ & $14,2 \%$ & $2,9 \%$ & $7,58 \%$ & $48,44 \%$ \\
\hline & Causes sociales & $15 \%$ & $9,1 \%$ & $1,4 \%$ & $8,66 \%$ & $28,13 \%$ \\
\hline
\end{tabular}




\section{Le marketing direct}

Le marketing direct est un outil utilisé par un site Web d'entreprise. Certains considèrent d'ailleurs qu'Internet est un formidable outil de marketing direct à lui seul, permettant la vente en ligne, la présentation d'un catalogue de produits, le publipostage, etc. et ce, à coût moindre par rapport aux mêmes activités réalisées avec les outils traditionnels. La définition générale du marketing direct retenue est la suivante : « The total of activities by witch the seller, in effectting the exchange of goods and services with the buyer, directs efforts to a target audience using one or more media (direct selling, direct mail, telemarketing, direct-action advertising, catalogue selling, cable TV selling, etc.) for the purpose of soliciting a response by phone, mail or personal visit from a prospect or customer $\gg{ }^{17}$.

Appliquée aux nouvelles technologies, cette définition du marketing direct inclut, entre autres, la vente en ligne, les circulaires électroniques, la notion de service à la clientèle, la présence d'adresses de courrier électronique ou encore l'existence d'un catalo- gue. Le tableau 3 résume les outils ainsi que leur degré d'adoption.

Encore une fois, on note une différence entre les études effectuées auprès des détaillants et celles des manufacturiers. Les premiers utilisent davantage les principaux outils tels que les catalogues, le courrier électronique et la mise en place d'un service à la clientèle. C'est avant tout leur activité qui les pousse à avoir recours à de telles pratiques, lesquelles sont le pendant de celles déployées dans la communication traditionnelle. La vente par catalogue est une forme de vente complémentaire, et le réseau Internet retrouve cet aspect. Toutefois, aucune des études mentionnées ici n'a pris en compte cette dimension. Le courrier électronique est le seul lien qui unit l'entreprise à son client pour une demande d'information, le règlement d'un litige, l'envoi d'une commande, etc. Quant au service à la clientèle, il se résume parfois, même sur un site Web, à un fiche préétablie ou un numéro de téléphone 1-800 pour rejoindre un préposé ! Il ne faut pas s'émerveiller devant un tel outil qui, bien souvent, n'est qu'un intermédiaire de plus entre le consommateur et l'entreprise.

\section{Tableau 3 - Synthèse des composantes du marketing direct sur les sites Web des entreprises nord-américaines}

\begin{tabular}{|c|c|c|c|c|c|c|c|}
\hline & Composantes & $\begin{array}{l}\text { Perry et } \\
\text { Bodkin } \\
(2003)\end{array}$ & $\begin{array}{l}\text { Perry et } \\
\text { Bodkin } \\
(2002)\end{array}$ & $\begin{array}{c}\text { Bégin et } \\
\text { Bois-vert } \\
(2002)\end{array}$ & $\begin{array}{c}\text { Bégin et } \\
\text { Tchokogué } \\
\text { (2001) }\end{array}$ & $\begin{array}{l}\text { Perry et } \\
\text { Bodkin } \\
(2000)\end{array}$ & $\begin{array}{c}\text { Griffith et } \\
\text { Krampf } \\
\text { (1998) }\end{array}$ \\
\hline \multirow{4}{*}{ 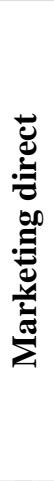 } & $\begin{array}{l}\text { Catalogue } \\
\text { électronique }\end{array}$ & $37 \%$ & $8,0 \%$ & $58,5 \%$ & $41,50 \%$ & $35,06 \%$ & n.é. \\
\hline & $\begin{array}{l}\text { Personnalisation } \\
\text { du service }\end{array}$ & n.é. & n.é. & n.é. & n.é. & $38,96 \%$ & $85,80 \%$ \\
\hline & $\begin{array}{l}\text { Adresses de cour- } \\
\text { rier électronique } \\
\text { (contactez-nous) }\end{array}$ & $70 \%$ & $19,1 \%$ & n.é. & $27 \%$ & $82,61 \%$ & $85,8 \%$ \\
\hline & $\begin{array}{l}\text { Service à la } \\
\text { clientèle }\end{array}$ & $22,0 \%$ & $8,0 \%$ & $49,7 \%$ & n.é. & n.é. & n.é. \\
\hline
\end{tabular}

Pourtant, en bénéficiant des enjeux de l'Internet, le marketing direct pourrait s'enorgueillir de devancer les autres formes de communication. Les nombreuses informations recueillies sur le client lors de son passage sur le site Web, de même que celles divulguées volontairement par le client lors de participation à des concours, lors de demande d'échantillons, etc. constituent des bases de données utilisables par l'entreprise à diverses fins.

En fonction de la valeur que l'on attribue à un client, la méthode des scoring (valeur) permet de filtrer les 
informations de la base de données et de consacrer plus ou moins de temps pour lui vendre un produit ou lui proposer un service, et l'investissement en temps, argent et hommes ne sera pas le même selon les clients considérés.

L'autre avantage est que cette technique s'apparente avant tout à du marketing stratégique et qu'elle permet de cibler les interlocuteurs clés d'une entreprise cliente. Ainsi, on peut s'adresser de façon différente et personnalisée au client, et surtout, l'entreprise y engage des efforts différents selon les clients : la relation est personnalisée. Ainsi, cette méthode s'applique tout aussi bien aux entreprises clientes qu'aux consommateurs finaux.

\section{La promotion des ventes}

Deuxième outil en importance en termes d'utilisation par les entreprises, la promotion des ventes a sa place sur Internet où les outils traditionnels tels que les coupons, les jeux concours, les rabais ou la démonstration virtuelle se conjuguent à merveille avec les nouvelles technologies. La promotion des ventes se définit par Desmet (1992) comme suit : «L'ensemble des actions marketing, ponctuelles et ciblées, caractérisées par une offre et les médias associés (en émission et en réception) visant à la création de nouveaux comportements actuels d'agents et d'intermédiaires au sens large (prescripteur, acheteur, vendeur) contri- buant à l'accroissement de la demande à l'entreprise $\gg{ }^{18}$.

Les buts de la promotion des ventes diffèrent toutefois quelque peu selon que l'on est une entreprise manufacturière ou un détaillant. Ainsi, pour ces derniers, les objectifs de la promotion des ventes consistent à distribuer de nouveaux produits, améliorer l'image de marque de produits existants, écouler des surplus d'inventaire ou mettre en valeur des marques existantes. Pour parvenir à ces fins, plusieurs outils sont utilisés, sur les deux types de communication, notamment les rabais, les jeux concours, le couponnage, les échantillons gratuits et les démonstrations en ligne pour la communication électronique. Cette composante non étudiée ici est l'apanage de certaines entreprises (Lands End) mettant en valeur leur avant-gardisme en utilisant les prouesses technologiques de certaines entreprises comme Mon Mannequin Virtuel (www. monmannequinvirtuel.com).

Pour les autres composantes, le caractère passager de cette activité rend difficile toute mesure, si bien que les résultats présentés dans le tableau 4 sont à prendre avec précaution. Les données présentées ici ne sont qu'une photo prise à un moment de l'année et ne reflètent pas forcément le recours permanent à ces outils de communication. Quoi qu'il en soit, les outils les moins utilisés restent les cadeaux ou échantillons gratuits et le couponnage.

\section{Tableau 4 - Synthèse des composantes de la promotion des ventes sur les sites Web des entreprises nord-américaines}

\begin{tabular}{|c|c|c|c|c|c|c|}
\hline & Composantes & $\begin{array}{c}\text { Perry et } \\
\text { Bodkin } \\
\text { (2003) }\end{array}$ & $\begin{array}{c}\text { Perry et } \\
\text { Bodkin } \\
(2002)\end{array}$ & $\begin{array}{c}\text { Bégin et } \\
\text { Boisvert } \\
\text { (2002) }\end{array}$ & $\begin{array}{c}\text { Perry et } \\
\text { Bodkin } \\
(2000)\end{array}$ & $\begin{array}{c}\text { Griffith et } \\
\text { Krampf } \\
\text { (1998) }\end{array}$ \\
\hline \multirow{4}{*}{ 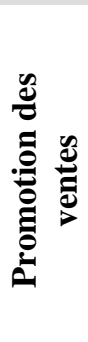 } & $\begin{array}{l}\text { Promotions } \\
\text { générales }\end{array}$ & $20 \%$ & $39,4 \%$ & $1,5 \%$ & $35,14 \%$ & $32,8 \%$ \\
\hline & $\begin{array}{l}\text { Cadeaux/échantil- } \\
\text { lons gratuits }\end{array}$ & $5,0 \%$ & $33,3 \%$ & $1,6 \%$ & $18,92 \%$ & $9,4 \%$ \\
\hline & Jeux concours & $13 \%$ & $24,2 \%$ & n.é. & $2,70 \%$ & $18,8 \%$ \\
\hline & Couponnage & $2,0 \%$ & $3,0 \%$ & n.é. & $2,70 \%$ & $9,4 \%$ \\
\hline
\end{tabular}

Pour les premiers, il semble difficile de les intégrer au site Web de l'entreprise : en fait il s'agit plus d'une rubrique visant à faire la demande d'un échantillon pour tester le produit. Nous considérons qu'il est peu 
utile sur un site Web de recourir à de telles pratiques, le consommateur ayant tout intérêt à aller se procurer le produit en question en magasin.

Pour le couponnage, ni le secteur d'activité ni le temps ne permettent d'expliquer ce peu d'intérêt des entreprises pour son utilisation. Dans la pratique, certaines grandes entreprises l'utilisent soit pour permettre aux clients d'avoir un rabais sur un produit de marque maison (www.selectionmerite.ca), soit pour lui donner des points boni dans un programme de fidélité (www.iga.net). Dans certains cas, les coupons sont même envoyés par la poste après une commande en ligne (www.save.ca de Procter \& Gamble). Il est probable que le peu d'intérêt de cet outil résulte aussi de la présence de sites Web spécialisés regroupant des coupons pour divers produits (www.publisac.ca, www.rabaistva.com, www.coupons.com,...).

\section{Les enjeux de la communication électronique pour les entreprises}

La panoplie d'outils de communication et de leurs composantes peut laisser bon nombre de dirigeants d'entreprise pantois, se demandant si leur site Web n'est pas désuet en raison du manque de certaines variables présentées ici. Il nous semble que la pénurie de ces outils n'engendre pas nécessairement des effets négatifs pour une entreprise.

Bien souvent, le choix de certains outils est influencé par les pratiques en vigueur au sein du secteur d'activité de l'entreprise. C'est d'ailleurs ce que les études américaines présentées ici démontrent. Dans un second temps, le choix des outils de communication est déterminé par la stratégie de communication ellemême. Elle peut d'ailleurs se décomposer en plusieurs étapes comme nous l'avons fait et, pour chacune d'elles, plusieurs questions doivent être posées pour être en mesure d'effectuer des choix :

- les cibles : le dirigeant doit se poser la question suivante: quels sont les acteurs avec qui l'entreprise cherche à entrer en communication ? À partir de ce moment, le choix des outils est en partie déterminé, et certains s'appliquent plus que d'autres. Les actionnaires seront intéressés par les rubriques des relations publiques; les consommateurs cher- cheront, par exemple, des circulaires électroniques ou des coupons rabais;

- le budget disponible : le coût de lancement d'un site Web peut être considérable mais, dans certains cas, l'entreprise peut être amenée à le gérer de façon autonome par la suite, ce qui est plutôt rare pour les outils de la communication traditionnelle;

- les objectifs de l'entreprise : ils renvoient à ce que les spécialistes du marketing nomment les modèles de hiérarchie des effets; pour chaque niveau de ces modèles, des gestes tactiques (outils) sont à poser pour attirer l'attention du consommateur, susciter son intérêt, attiser son désir ou encore le pousser à l'action.

Les entreprises qui respectent ces étapes pourront mieux bâtir leur stratégie de communication, qu'elle soit traditionnelle ou électronique, et les choix opérés seront adéquats et pertinents. Cette forme d'intégration des communications traditionnelles et électroniques est plus que jamais vraie dans un environnement où l'on constate une multiplicité des outils de communication, une évolution rapide des nouvelles technologies amenant leur lot de nouvelles façons de communiquer, une augmentation de la concurrence et une abondance d'informations à la disposition des clients.

Il nous semble également que la communication soit une variable centrale du cybermarketing mix, et l'intégration des autres (produit, prix, et distribution) est nécessaire, car elles se font toutes écho dans la communication. À titre d'exemple, disons que le produit est présent sur les circulaires, dans les catalogues et les sites Web, le prix est annoncé dans les circulaires et la distribution peut se faire de façon électronique par le biais du site Web, un outil basique de la cybercommunication marketing.

\section{Notes et références}

1 William Menvielle, Denis Pettigrew et Jocelyn D. Perreault sont tous trois professeurs de marketing à l'UQTR.

2 Voir Menvielle, W., D. Pettigrew et J.D. Perreault (2002). «Le cyber-produit comme conjugaison du produit au monde virtuel: une génération de nouvelle valeur ajoutée ", revue Organisations et territoires, vol. 11, $\mathrm{n}^{\circ} 2$, printemps-été; Menvielle, W., D. Pettigrew et J.D. Perreault (2002). «Le cyber-prix : une adéquation nécessaire 
avec les modèles d'affaires de l'Internet », revue Organisations et territoires, vol. 11, n ${ }^{\circ}$, printemps-été; Menvielle, W., D. Pettigrew et J.D. Perreault (2003), « La cyber-distribution : enjeux et tendance », revue Organisations et territoires, vol. $12, \mathrm{n}^{\circ} 2$, printemps-été.

3 Voir Pettigrew, D. et N. Turgeon (2004). Marketing, $5^{\mathrm{e}}$ édition, Montréal, Chenelière McGraw-Hill.

4 Lambin, J-J. (1998). Le marketing stratégique: une perspective européenne, $4^{\mathrm{e}}$ édition, Paris, Ediscience international.

5 Schultz, D.E. (1993). « Integrated Marketing Communications : Maybe Definition is in the Point of View », Marketing News, January, vol. 27, $\mathrm{n}^{\circ} 2$, p. 17-18.

6 Kotler, P., P. Filiatrault et E. Turner (2000). Le management du Marketing, $2^{\mathrm{e}}$ édition, Boucherville (Québec), Gaétan Morin; Schultz, D.E. (1993), op. cit.; Belch, G.E., M.A. Belch et M. Guolla (2003). Advertising and Promotion, Toronto, McGraw-Hill Irwin.

7 Ritson, M. (1998). « Three Possibilities in Integrating the Internet ». Integrated Marketing, May, vol. 11, p. 10-12.

8 Griffith, D.A. et R.F. Krampf (1998). «An Examination of the Webbased Strategies of the Top 100 US Retailers », Journal of Marketing Theory and Practice, Summer, p. 12-23.

9 Perry, M. et C. Bodkin (2000). « Content Analysis of Fortune 100 Company Web Sites », Corporate Communications: An International Journal, vol. 5, n ${ }^{\circ}$ 2, p. 87-96; Perry, M. et C. Bodkin (2002). « Fortune 500 Manufacturer Web Sites : Innovative Marketing Strategies or Cyberbrochures ? », Industrial Marketing Management, vol. 31, p. 133-144; Perry, M. et C. Bodkin (2003). « Goods Retailers and Service Providers : Comparative Analysis of Web
Site Marketing Communications », Journal of Retailing and Consumer Services.

10 Bégin, L. et A. Tchokogué (2001). Étude sur le profil des sites Web des entreprises québécoises, Cahier de recherche CICMA 01-02, Montréal, École des HEC; Bégin, L., A. Tchokogué et H. Boisvert (2001). Pour un déploiement stratégique du commerce électronique, Saint-Hyacinthe (Québec), Isabelle Quentin Éditeur.

11 Décaudin, J-M. (1999). La Communication Marketing, concepts, techniques, stratégies, $2^{\mathrm{e}}$ édition, Paris, Économica.

12 Bourliataux-Lajoinie, S. (2000). Personnalisation de la publicité sur Internet dans un contexte international: une méthode efficace ?, colloque international «Évolution du marketing dans le monde arabe : apport des technologies de l'information », Beyrouth, Université Saint-Joseph, 2 et 3 novembre 2000 , http://www.usj.edu.lb/colloques/fgm1/fgm1bou.htm

13 Dans tous les tableaux, n.é. signifie « non évalué ».

14 Sfez, L. (1992). Critique de la communication, Paris, Points, Seuil.

15 Il s'agit ici des variables : « Histoire de la compagnie », « Mot du président », « Ce que nous faisons ».

16 Kotler, P. (1999). Le Marketing selon Kotler, Paris, Éditions Village Mondial.

17 Belch, G.E. et M.A. Belch (2001). Advertising and Promotion, Toronto, McGraw-Hill Irwin.

18 Desmet, P. (1992). Promotion des ventes, du 13 à la douzaine au marketing direct, Paris, Nathan. 


\section{PUBLICITÉ}

Études de cycles supérieurs 